\title{
Measuring the Relative Efficiency of Government Banks Using Data Envelope Analysis (DEA) For The Period (2010-2019)
}

\author{
Ali Hamed Abd*, Mohammed Jassim Ahmed \\ Department of Economics, College of Administration and Economics, University of Fallujah, Iraq \\ *cae.aha@uofallujah.edu.iq
}

\begin{abstract}
KEYWORDS: $\quad$ Measurement, Data Envelope, Analysis, Efficiency, Government Banks.
\end{abstract}
\section{ABSTRACT:}

The research aims to apply the data envelope analysis method to measure the efficiency of Iraqi government banks for the period (2010-2019) and to implement the objectives of the research, the method of data envelope analysis was chosen on (7) government banks for the period (2010-2019), this research means measuring the efficiency of the Iraqi banking system The research reached a main conclusion that (6) banks did not achieve efficiency, namely (Al-Rafidain Bank, Al-Rasheed Bank, Agricultural Bank, Industrial Bank, Real Estate Bank, Trade Bank of Iraq) and one bank that achieved efficiency during the period 2010-2019, which is (Al-Nahrain Bank Islamic). Therefore, the research recommends Iraqi government banks to work to raise their efficiency by modernizing banks to strengthen their financial positions, and to provide new banking services to face intense competition in light of the liberalization of banking services trade, and the research recommends the necessity of financing provided by government banks to achieve economic development.

\section{REFERENCES:}

Alex Manzoni and Sardar lslam (2009) Performance Measurement in Corporate Governance: DEA Modelling and Implications for Organisational Behaviour and Supply Chain Management، Springer Science Business Media, physica-Verlag Heidelberg, Germany.

Avkiran, Neei (1999) an Application Reference for Data Envelopment Analysis in Pranch Banking: Helping the Novice Research. International Journal of Bank Marketing.

Bauer,p.w .A.N. Berger and D .B. Humphrey (1993) Efficiency and Productivity Growth in U.S Banking. In H. Fried C.A.K. Lovell, and P. Schmidt (Eds) The Measurement of Productive Efficiency: Techniques and Applications, Oxford: Oxford University press.

Charnes, A, Cooper (1994), W, Lewin, A. and Seiford, L(eds) Data Envelopment Analysis-theory, Methodology and Applications Kluwer Academic publishers.

Coelli, T, Rao, D. and Battese, G. (2005) An Introduction to Efficiency and Productivity Analysis. Springer Science + Business Media, lnc. New York

Emmanuel Thanassoulis, Maria C.S. Portela and Ozren Despic (2008) Data Envelopment Analysis: The Mathematical Programming Approach to Efficiency Analysis in the Measurement of Productive Efficiency and Productivity Growth Edited by Harold O. Fried C.A knox Lovell and Shelton S. Schmidt, Oxford University Press, Inc. New York

Fliabetta Fiorentino, Alexander Karmann, and Michael Koetter (2006) The Cost efficiency of German banks: a comparison of SFA and DEA ,Deutsche Bundesbank ,Discussion paper, Series 2: Banking and Financial Studies.

Rouatt Stephen (2003) Two stage evaluation of bank branch efficiency using data envelopment analysis. Unpublished University of Toronto. USA.

Sarafidis, V. (2002) An Assessment of Comparative Efficiency Measurement Techniques, Europe Economics, office of Water Services

Rowena Jacobs, peter C. Smith and Andrew Street (2006) Measuring Efficiency in Health Care: Analytic Techniques and Health Policy, Cambridge University Press, New York. 
قياس الكفاءة النسبية للمصارف الحكومية باستخدام تحليل مغلف البيانات (DEA) للمدة (2019-2010)

\author{
علي حامد عبد.*، أ.م.د. احمد محمد جاسم \\ قسم الاقتصاد، كلية الإدارة والاقتصاد، جامعة الفلوجة، العراق \\ *cae.aha@uofallujah.edu.iq
}

قياس، مغلف البيانات، تحليل، كفاءة، المصارف الحكومية.

الكلمات المفتاحية

Crossref d

https://doi.org/10.51345/.v32i4.436.g245

ملخص البحث:

يهدف البحث إلى تطبيق أسلوب تحليل مغلف البيانات لقياس كفاءة المصارف العراقية الحكومية للمدة (2010-2019)

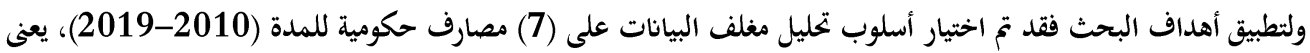

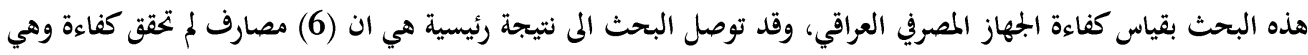

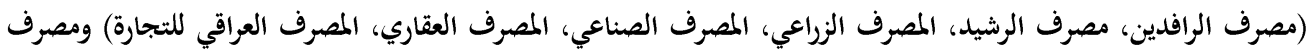

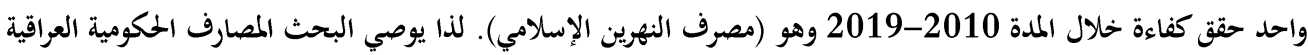

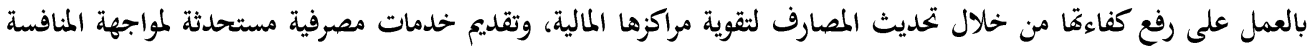

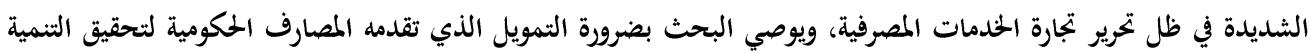
الاقتصادية.

المقدمة:

يعتمد نجاح أية مؤسسة اقتصادية على القدرة في اتخاذ القرارات الصحيحة بشكل أساسي في الأوقات

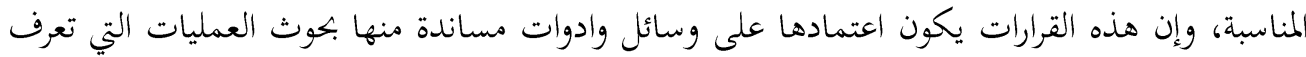
بأها عملية لصنع القرار المبنية على المنهج العلمي والتي يكون اعتمادها بصفة أساسية على التحليل الكمي لماتي لحل المشكلات الاقتصادية للوصول الى البديل الأمثل في حدود الامكانات المتاحة والمجالات التي يمكن أن تسهم فيها بكوث العمليات والمتعلقة باقتصاديات الانتاج لاستخدام الموارد المالية والبشرية والتكنلوجية للحصول على مستويات عالية من الانتاج. 
وفي عام 1978 ظهر أسلوب جديد لقياس الكفاءة النسبية للمؤسسات وهو أسلوب تحليل مغلف

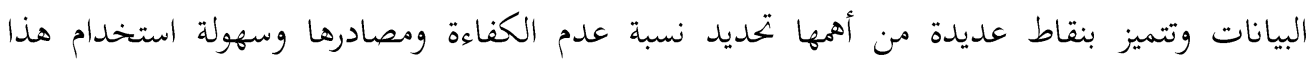

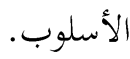

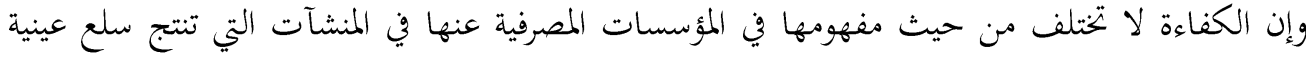

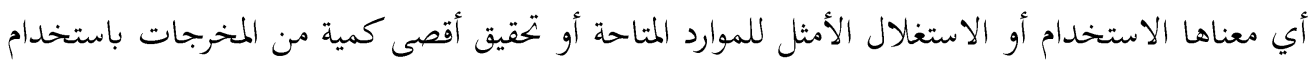

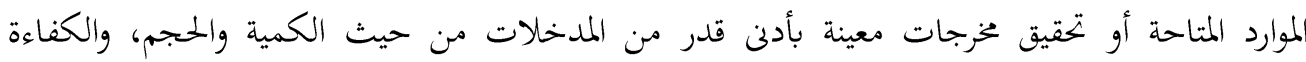
المصرفية تتمثل بالعلاقة بين مدخلات المصرف ومخرجاته وقياس كفاءة المصارف تعد من أهم العناصر الرئيسة لنجاحها، وظهر هذا الاهتمام بقياس الكفاءة عن طريق استخدام بجموعة من الأساليب التقليدية

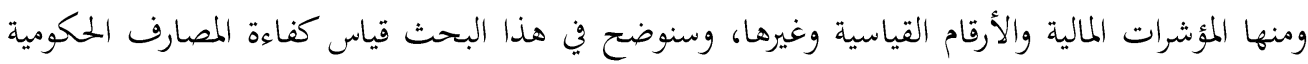
للمدة 2010-2019 باستخدام مغلف البيانات.

\section{أهمية البحث:}

تتزايد اهمية قياس الكفاءة في بجال النشاط المصريف للمصارف الحكومية انطلاقا من ضخامة الأموال التي

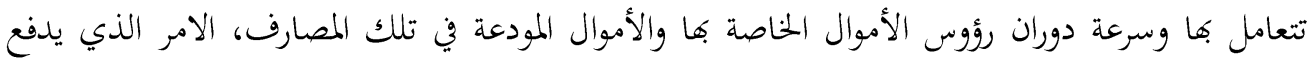

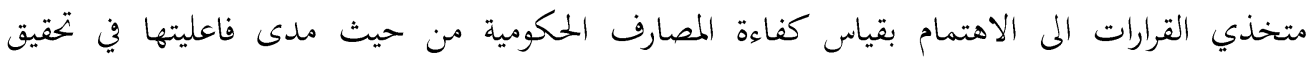

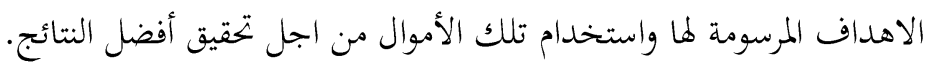

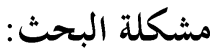

تتجسد مشكلة البحث في ضعف الاداء المصري في العراق رغم الزيادات المتلاحقة في عدد المصارف مما

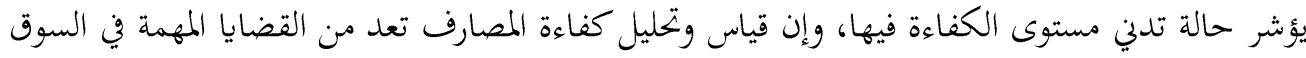

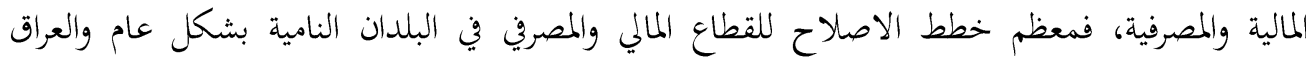
بشكل خاص تركز على تحسين كفاءة عمل المصارف من خلال الاهتمام بدور المؤسسات المصرفية وزيادة المنافسة في السوق المالية، وإن التوجهات الجديدة للسياسة الاقتصادية في العراق بعد عام (2003)

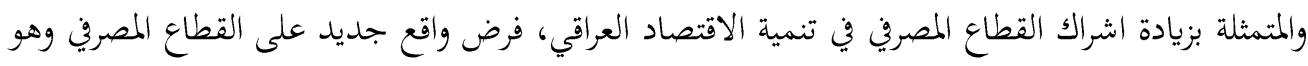
أن يأخذ دوراً اكبر في النشاط الاقتصادي وفي دعم الاقتصاد وتمويل مشاريع التنمية الاقتصادية. 


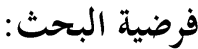

للمصارف الحكومية دور إيجابي في دعم وتمويل القطاعات الاقتصادية المختلفة من خلال تسهيل اجراءات حصول هذه القطاعات على القروض والتسهيلات الائتمانية على افتراض أن القطاعات الاقتصادية تؤدي إني دوراً كبيراً في التنمية الاقتصادية والاجتماعية.

هدف البحث:

يهدف البحث الى قياس مستوى الكفاءة التي تعمل فيها وحدات الجهاز المصرفي العراقي خلال المدة

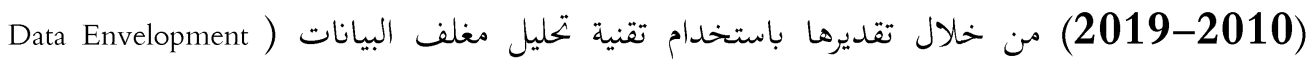
ومن خلال هذا التقدير يتم تحديد المصارف الحكومية العاملة في مستوى الكفاءة التامة (DEA) (Analysis في القطاع المصرين العراقي، فضلا عن تحديدها كمصارف مرجعية للمصارف غير الكفوءة.

\section{منهجية البحث:}

تم الاعتماد على المنهج النظري والكمي في اختبار فرضية البحث وتحقيق اهدافه، ولاختبار فرضيات البحث

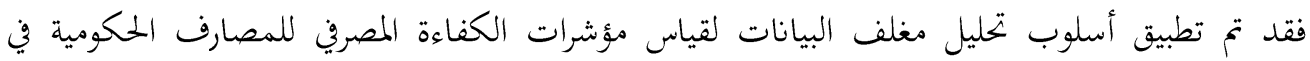
العراق، وقد تم جمع بيانات عن (7) مصارف حكومية للمدة (2010-2019) وذلك بسبب توافر البيانات اللازمة لتطبيق (أسلوب تحليل مغلف البيانات DEA) على المصارف الحكومية، فضلا عن الاعتماد على التقارير والنشرات الاحصائية السنوية للبنك المركزي العراقي، دائرة الاحصاء والابحاث في جمع البيانات.

\section{حدود البحث:}

1-الحدود المكانية: تتمثل الحدود المكانية للبحث بدراسة كفاءة المصارف الحكومية في العراق. 2-الحدود الزمانية: تتمثل الحدود الزمانية للبحث بالمدة (2010-2019).

\section{هيكلية البحث:}

يتضمن المبحث الاول: قياس كفاءة المصارف الحكومية للمدة (2010- 2019) ويتضمن المبحث الثاني: خطوات تطبيق أنموذج (DEA) على المصارف الحكومية. 


\section{المبحث الاول: قياس كفاءة المصارف الحكومية}

\section{طرق قياس الكفاءة المصرفية:}

اسلوب تحليل مغلف البيانات (Data Envelopment Analysis DEA) يعد تحليل مغلف البيانات أحد أهم أدوات الأسلوب اللامعلمي الذي يستخدم أسلوب البربجة الخطية لقياس الكفاءة النسبية لمجموعة منشآت والتي تسمى وحدات (اتخاذ القرار)، وتم تطويره في البداية لتقييم الكفاءة النسبية لوحدات المنظمة الواحدة مثل فروع المصرف الواحد، وتكون وحدات إتخاذ القرار متجانسة من حيث استخدام نوعية متشابهة من المدخلات والمخرجات، ليبين كيفية الحفاظ على موارد تلك الوحدة

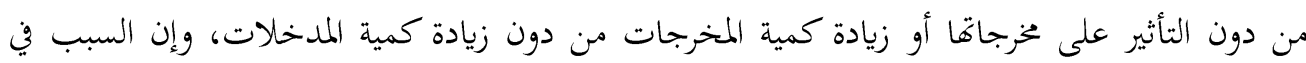
تسمية مغلف البيانات ههذا الاسم يرجع الى الشكل الذي تتخذه حدود الكفاءة، والوحدات الإدارية التي

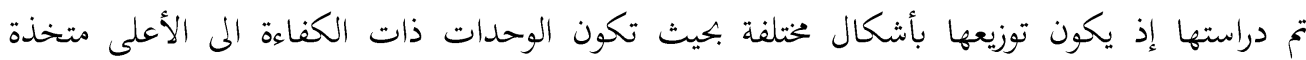
منحنى مقعر باتجاه نقطة الأصل وتغلف الوحدات الأقل كفاءة، ويمكن قياس عدم الكفاءة من خلال المسافة الفاصلة بينها وبين المنحنى الذي يمثل الوحدة الكفؤة (منصوري، 2014: 82) ويكون اشتقاق الكفاءة من خلال مجموعة من المؤسسات التي تشكل المنحنى الحدودي للأداء والذي يغلف كل المشاهدات، وتتمتع المؤسسات التي تقع على المنحنى الحدودي بالكفاءة في عملية التوزيع لمدخلاتما وإنتاج مخرجاتها، أما المؤسسات التي لا تقع على المنحنى الحدودي غير كفوءة أي إن اسلوب مغلف لماتى البيانات يقوم

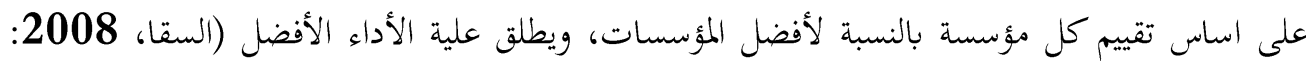
42) وإن أسلوب تحليل مغلف البيانات يعتمد على أمثلية (باريتو) والتي تنص على أن "أي مؤسسة اعمال تكون غير كفوءة إذا استطاعت أي مؤسسة اخرى أو مزيج من مؤسسات الاعمال الأخرى إنتاج نفس

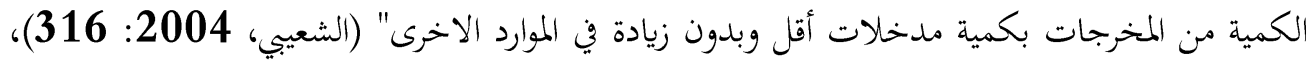
ويقدم تحليل مغلف البيانات معلومات إضافية مفيدة للتعرف على أداء وتوجيه هذه المؤسسات لتحسين أدائها، ويقدم مفهوم مؤسسات مناظرة لكل مؤسسة غير كفوءة وتعد ميزة لمغلف البيانات ويمكن أن تكون

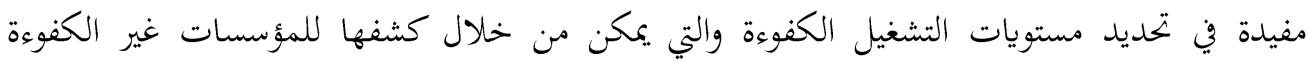
لتحسين الأداء (Emmanuel et al 2008) 252)، وان مغلف البيانات يساهم عند تقييم اداء مجموعة من المؤسسات في تحديد مؤشرات مهمة لمؤسسات الاعمال وهي كالاتي (العزاز، 2000: 20- 26): أ- تحديد المؤسسات التي تكون كفاءقّا تامة: يؤدي التعرف على الممارسات التشغيلية عند المؤسسات

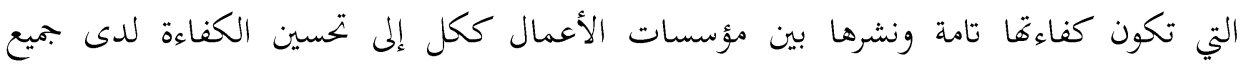


مؤسسات الأعمال الاخرى التي تعاني ضعفا في أدائها وليس فقط على مستوى المؤسسة نفسها، ومؤسسات الأعمال التي تكون كفاءقا تامة هي المصدر الأساسي للممارسات التشغيلية الناجحة. ب-تحديد المؤسسات المرجعية: تعد كل مؤسسة أعمال مرجعية ذات كفاءة تامة عند استخدام الأوزان الخاصة بتلك المؤسسة التي ليست ذات كفاءة تامة، وإن أسلوب مغلف البيانات يقدم لكل مؤسسة موسية أعمال ليست كفاءتا تامة، مجموعة من مؤسسات الأعمال التي تكون كفاءتها تسمى مؤسسة مرجعية

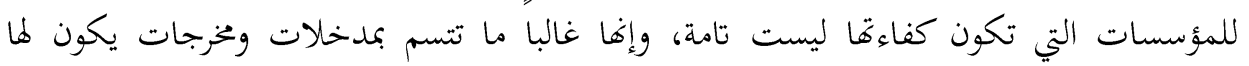
تأثيرات متماثلة في حساب الكفاءة، ويمكن استخدامها لتقدم أمثلة عن الممارسات المتميزة التي يمكن أن تدرسها مؤسسات الأعمال التي تكون كفاءها ليست تامة وتعمل على محاكاتها لتقوم بتحسين مستويات الكفاءة، ويمكن للمؤسسات المرجعية أن تقدم اهداف ملائمة للمؤسسات التي تكون كفاءقا غير تامة. ج- تخصيص الموارد: إن تحليل مغلف البيانات يقدم تقديرا لإمكانات ترشيد الموارد وتحسين مستويات كاءتهات المخرجات عند المؤسسات التي تكون ذات كفاءة منخفضة، وتقوم بمساندة متخذ القرار في تخصيص لماتيل الموارد لمؤسسات الاعمال. د- وضع الأهداف التخطيطية: تكون رغبة متخذ القرار في وضع أهداف لرفع مستوى المخرجات فقط أو يرغب في تحديد موازنة لخفض مستوى المدخلات فقط، أو يقوم برفع مستوى المخرجات وخفض رهض

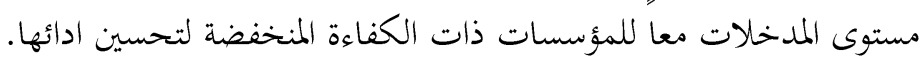
وتصنف المؤسسات على أساس مستويات الكفاءة المحققة أفضل أداء بين مفردات المجموعة يجصل على ملى مأل قيمة تساوي (1)، أما المؤسسات ذات الكفاءة الأقل فتحصل على قيم اقل من الواحد، وكلما الخغض إنض القياس المحسوب للمؤسسة كلما الخخضت كفاءة هذه المؤسسات. وان مغلف البيانات يتطلب توافر ثلاثة متطلبات اساسية وهي:(Rouatt,2003:25).

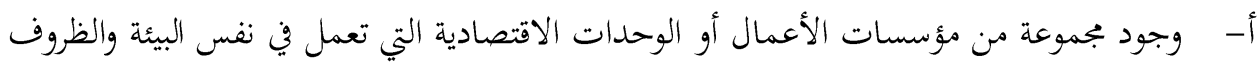

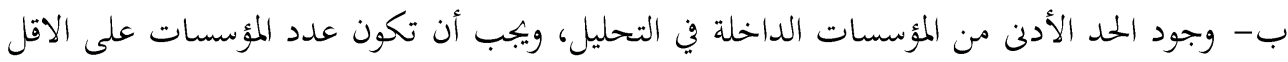
ثلاثة اضعاف مجموع المدخلات والمخرجات، الامر الذي يعطي درجة حرية كافية عند تطبيق هذا الأسلوب لقياس الكفاءة. ج- ضرورة وجود مجموعة من البيانات لجميع مدخلات ومخرجات المؤسسات الداخلة في عملية التحليل 
ولتوضيح تحليل مغلف البيانات نستعين بالشكل (1-1) ونفترض وجود أربع مؤسسات أعمال وهي (B-C-D A-) تحسب الكفاءة لكل مؤسسة اعمال مقارنة بكل المؤسسات الأخرى في المجموعة، اي أن المؤسسات احتهات (B-C

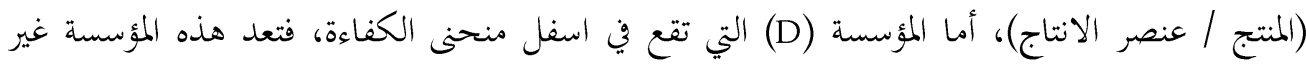
كفوءة لاها استخدمت كمية أكبر من المدخل لانتاج نفس المنتج للمؤسسات الاخرى، ويمكن لهذه المؤسسة أن تصبح كفوءة في حالتين: أ- أن تقوم المؤسسة بتخفيض استخدام المدخل مع الحفاظ على نفس المستوى من الانتاج (كما هو موضح بالنقطة (D/). ب-أن تقوم المؤسسة بزيادة انتاجها مع الحفاظ على نفس مستوى المدخل (كما هو موضح بالنقطة .(D// الشكل (1-1) : منحنى مغلف البيانات لعدد من المؤمسات

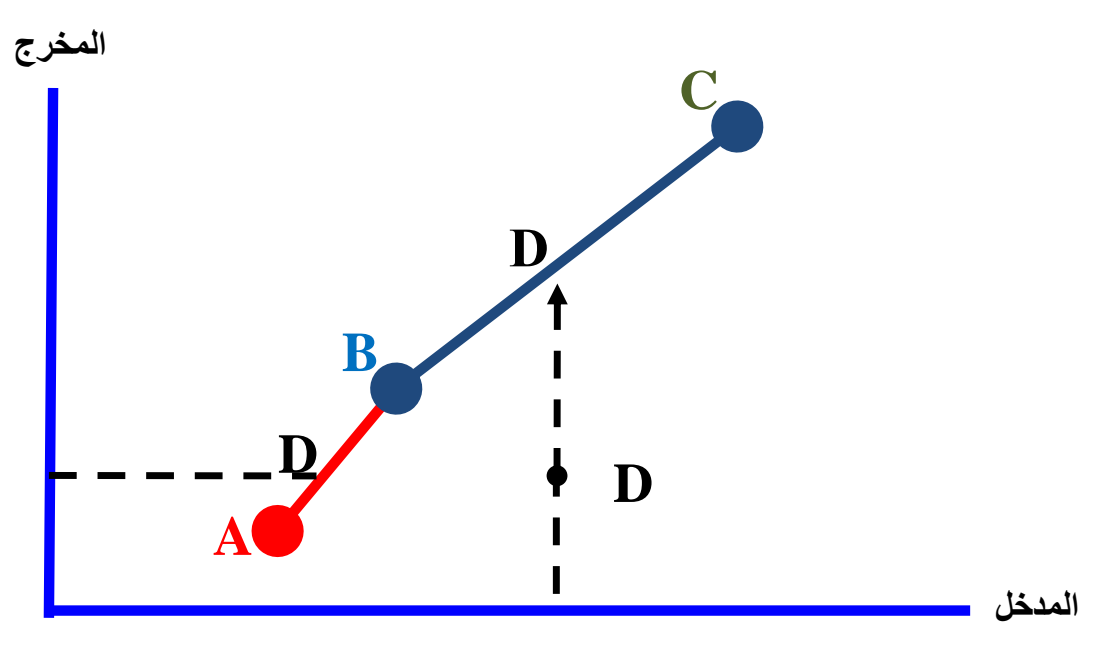

المصدر : بتال، احمد حسين، قياس وتحليل كفاءة أداء المصارف الخاصة في العراق باستخدام تكنيك مغلف البيانات، اطروحة دكتوراه مقدمة الى مجلس كلية الادارة والاقتصاد، جامعة بغداد، ص 98. 
ونلاحظ أن الخاصية المهمة لأسلوب مغلف البيانات هي إمكانية تحديد مستويات التحسين المطلوبة للمدخلات والمخرجات وتحديد المؤسسات المرجعية في المؤسسات التي تكون غير كفوءة، ويتم ذلك من خلال إسقاط كل مؤسسة غير كفوةة على منحى الكفاءة الحدودي ومن ثم يمكن تحديد مستويات

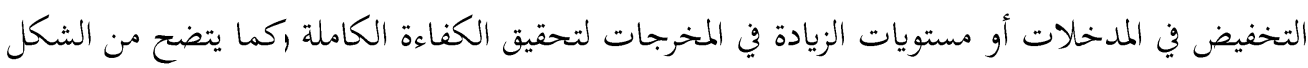

التحليل الحدودي العشوائي (Stochastic Frontier Analysis SFA)

Aigner,et تعد طريقة التحليل الحدودي العشوائي من الطرق المعلمية القياسية التي قدمت من قبل (اجنر)

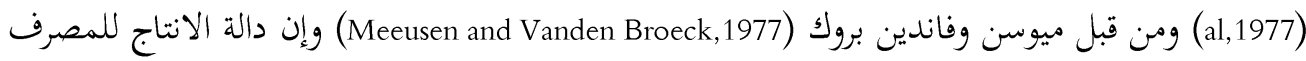
تكون وفق التحليل الحدودي العشوائي وفق الصيغة الاتية، (Flisabetta et al,2006:7):

$\operatorname{Ln}_{\mathrm{i}}=\operatorname{Ln} X_{\mathrm{i}} \beta+\mathrm{v}_{\mathrm{i}}-\mathrm{u}_{\mathrm{i}}$

$$
\begin{aligned}
& \text { LnYi } \\
& \text { Xi } \\
& \text { ק: يمثل المعلمات المقدرة } \\
& \text { يمثل الخطأ العشوائي vis }
\end{aligned}
$$

un

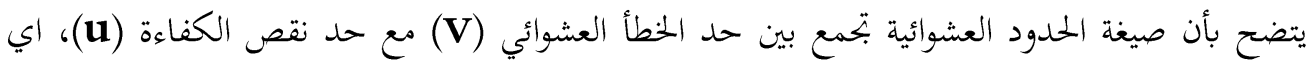

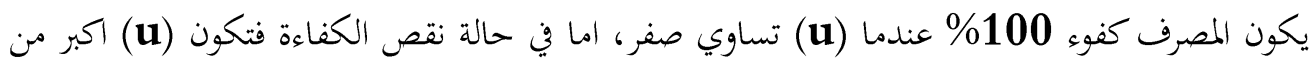
الصفر. ونفترض بأن الخطأ العشوائي يمثل خطأ القياس والاخطاء العشوائية الاخرى وان هذه الصيغة تفترض الاتي: .(Bauer etal 1993:287)

أ-عدم وجود اي ارتباط جطي بين الخطأ العشوائي وبين المتغير العشوائي. ب-أن المتغير العشوائي يكون توزيعه نصف طبيعي، لكون قيمة المتغير العشوائي اي (نقص الكفاءة) لا لا تأخذ قيمة غير سالبة. ج-يكون توزيع الخطأ العشوائي توزيعاً طبيعياً اي بمتوسط حسابي يساوي صفراً ويكون التباين ثابت أي ان . $\mathrm{N} \approx(0, \sigma 2)$ 
ويمكن توضيح صيغة الحدود العشوائي من خلال الرسم البياني، ونفترض لدينا بمموعة من المصارف تستخدم عنصر انتاجي واحد وتحقق منتج واحد، وتكون دالة الانتاج وفق الاتي:

$\mathrm{Y}_{\mathrm{i}}=\exp \left(\beta_{0}+\beta_{1} \operatorname{Ln} X_{\mathrm{i}}+\mathrm{v}_{\mathrm{i}}-\mathbf{u}_{\mathrm{i}}\right)$

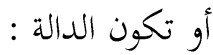

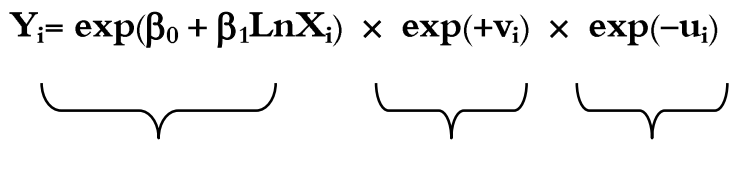

نقص الكفاءة الخطأ العشوائي المكون غير العشوائي

الشكل (1-2) التحليل الحدودي العشوائي

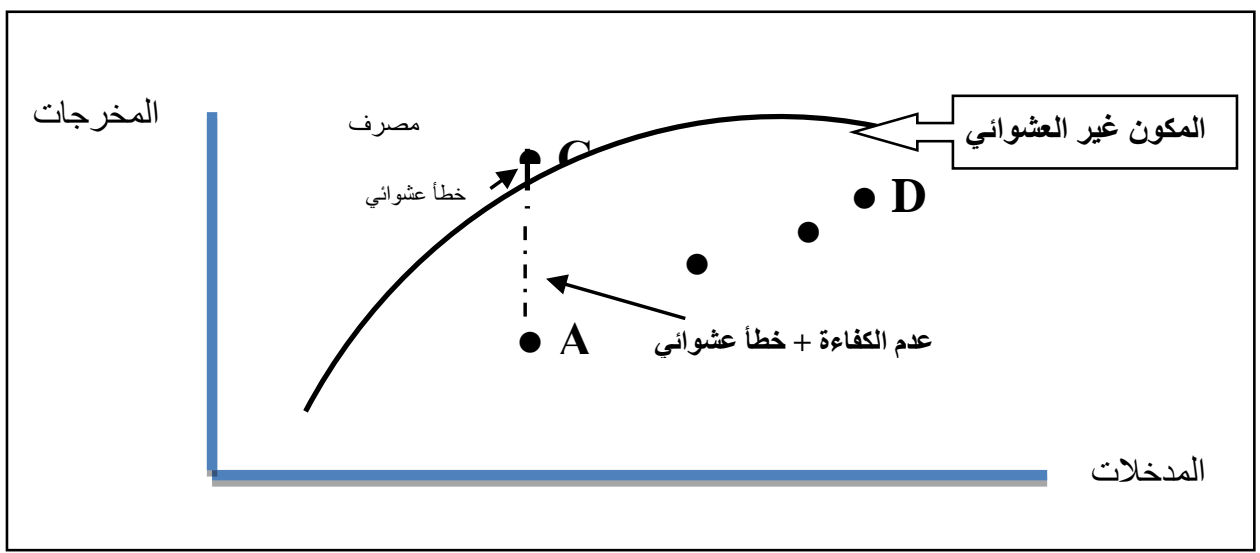

Bauer, P. W., A. N. Berger, G. D. Ferrier, and D. B. Humphrey (1998). Consistency Conditions for Regulatory Analysis of Financial Institutions: A Comparison of Frontier Efficiency Methods. Journal of Economics and Business No.50, p 95.

يتضح من الشكل (1-2) بأن المصرف (C) هو مصرف كفوء والذي يقع في أعلى منحنى الكفاءة الحدودي، والناتج عن الخطأ العشوائي، أما بالنسبة للمصارف الواقعة أسفل منحنى الكفاءة الحدودي تكون مصارف غير كفوءة أي عدم كفاءة المصرف مما يعني نقص الكفاءة الناتج من الخطأ العشوائي، وتوجد ميزات وسلبيات لنموذج التحليل الحدودي العشوائي (SFA) وهي: (Sarafidis,2002:9). 


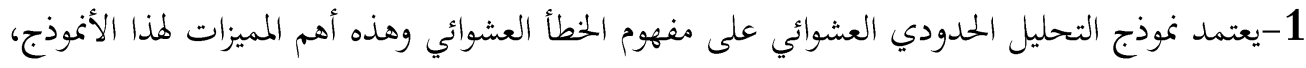
وأن هدف هذا التحليل هو فصل مكونات الخطأ عن حالة نقص الكفاءة، أما في حالة الجانب التطبيقي لا ينجح هذا الافتراض دائماً لكون تقدير نقص الكفاءة يمثل جزءاً من إجمالي تشتت البواقي أنموذج التحليل الحدودي العشوائي، وبسبب هذه الميزة المذكورة أعلاه فإن التحليل الحدودي العشوائي يكون مرتبطا بمفهوم إنهاء

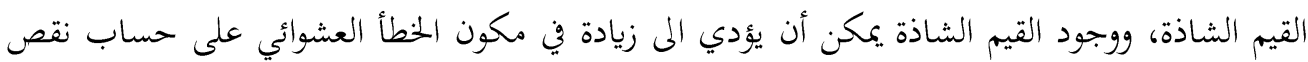

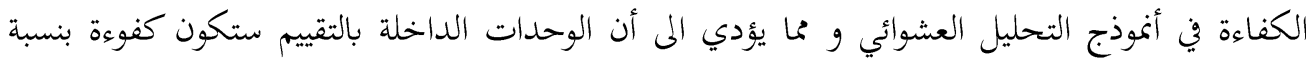

2-يخضع أسلوب التحليل الحدودي العشوائي لقيود نظرية، وإن أنموذج الكفاءة الحدودي العشوائي يحاول أن يقدر الكفاءة الفنية لجموعة من الوحدات المدروسة من خلال التمييز بين الخطأ العشوائي الإحصائي ونقص الكفاءة في بيانات العينة، مما يتطلب افتراض وجود توزيع معين للصيغة الدالية لحالة نقص الكفاءة ومن هذه التوزيعات التوزيع الأسي والتوزيع نصف الطبيعي. 3-يعطي أسلوب التحليل الحدودي العشوائي إستدلالا إحصائيا للأنموذج الحمدودي الدالي للكفاءة ويعطي

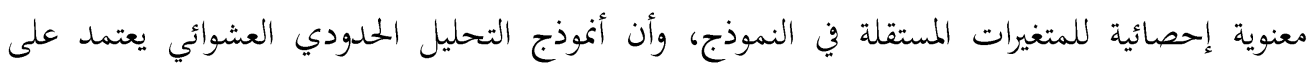

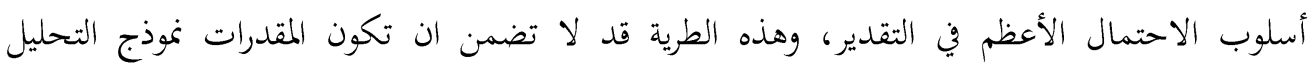
الحدودي العشوائي تحقق أي خصائص احصائية مرغوبة مثل (الكفاءة، عدم التحيز، الاتساق) للعينات الصغيرة للأنموذج. الكفاءة وتحليل مغلف البيانات إن الكفاءة تستند حسب مفهوم تحليل مغلف البيانات إلى مقالة (فاريل) عام 1957، إذ بين فاريل أن الكفاءة الاقتصادية تتضمن الكفاءة الفنية والكفاءة التوظيفية، وبين فاريل بأن هناك طريقتان لحساب الكابل مؤشرات الكفاءة الفنية، الأولى المؤشرات ذات التوجية الادخالي (oriented measures)، والثانية المؤشرات ذات التوجيه الاخراجي (output-oriented measures). المؤشرات ذات التوجيه الادخالي يتضح من الشكل (1-3) دالة الانتاج من وجهة الاستخدام لعدد من المؤسسات التي تنتج المخرج (y) وتستخدم (X1) و (X2) مدخل للانتاج تحت ظروف تتميز بثبات عائد الحجم. 


$$
\text { الشكل (1-3) أنموذج تحليل مغلف البيانات الادخالي }
$$

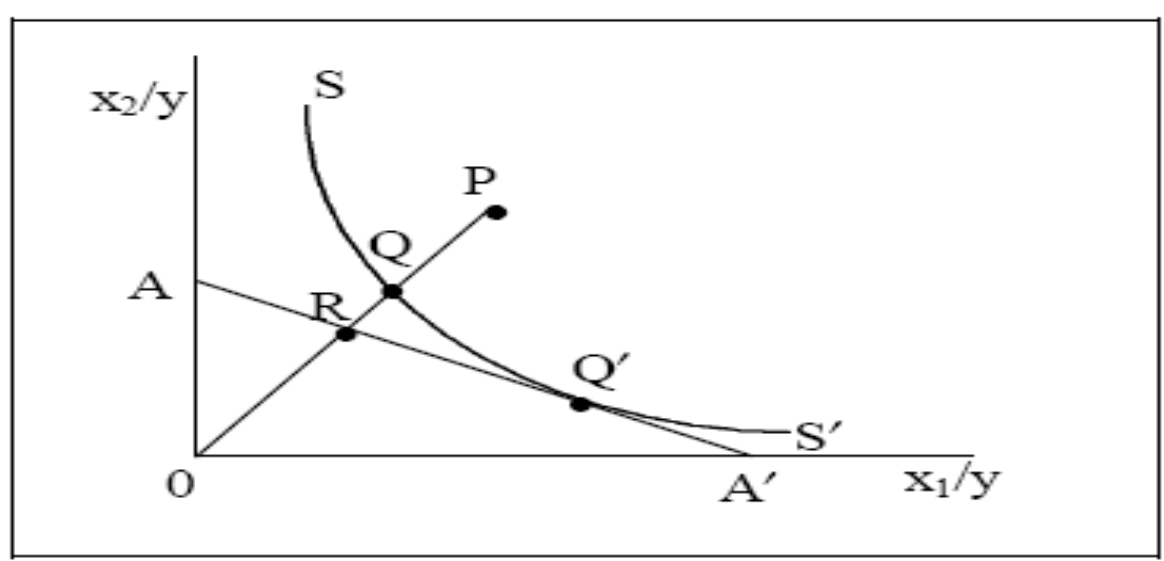

Alex Manzoni and Sardar Islam (2009) Performance Measurement in Corporate Governance: DEA Modelling and implications for Organizational Behaviour and Supply Chain Management, Springer Science Business Media, physica-Verlag Heidelberg, Germany.P.91.

بالنسبة ل (SS') تمثل انتاج وحدة واحدة من (y) بأقصى كفاءة باستخدام المدخلات (X1) و (X2)

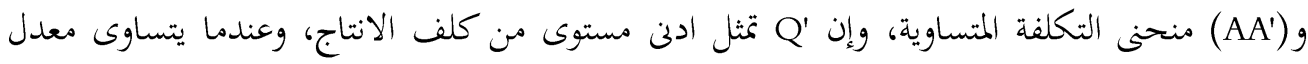
الاحلال الحدي بين المدخلين (X1) و(X2) مع نسب الاسعار، في هذه الحالة ان المؤسسة (p) تعد أقل مئل كفاءة من المؤسسة (Q) لانتاج وحدة واحدة، وإن المسافة (PQ) تعبر عن مدى الانخفاض في الكفاءة

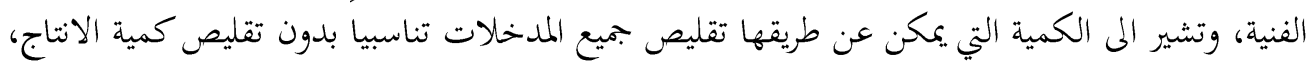
ومؤشر الكفاءة الفنية للمؤسسة التي تنتج عند النقطة (P) على المسار (OP) يكون وفق الصيغة الاتية: $T E=\frac{O Q}{O P}$

و يأخذ المؤشر القيم(-0) والقيمة (1) تدل على الكفاءة الفنية الكاملة للمؤسسة. أما ميل المستقيم (AA') 1 يمثل السعر النسبي للمدخلات وبمعرفة هذا الميل يمكن حساب مؤشر الكفاءة التوظيفية للمؤسسة على المستقيم (OP) بالصيغة الاتية: $A E_{i}=\frac{O R}{O Q}$ 
وان المسافة RQ تمثل المقدار الذي يمكن عن طريقه تخفيض كلفة إنتاج الوحدة من (y) بتوظيف المدخلات حسب المؤسسة (Q') بدلا عن المؤسسة (Q). المؤشرات ذات التوجيه الاخراجي ان الكفاءة من جانب المخرجات هي الكمية التي يمكن من خلالها زيادة المخرجات تناسبيا بدون تقليص

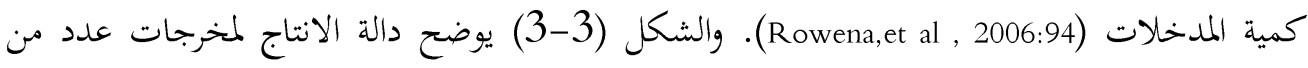
المؤسسات والتي تنتج نوعين من المخرجات (y1) و و(y2) وتستخدم مدخل الانتاج (X1) بظروف تتميز بثبات عائد الحجم.

الشكل (1-4) أنموذج تحليل مغلف البيانات الاخراجي

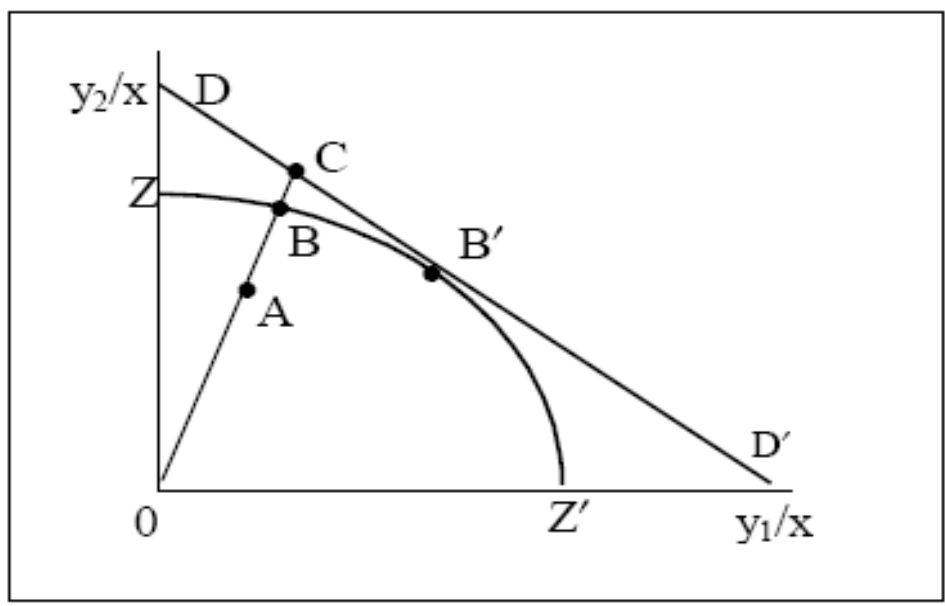

Alex Manzoni and Sardar Islam (2009)Performance Measurement in Corporate Governance: DEA Modeling and Implications for Organizational Behavior and Supply Chain Management, Springer Science+Business Media, Physica-Verlag Heidelberg, Germany. P 92

يمثل (zz') منحنى امكانات الانتاج ويمثل(DD') خط تساوي الايرادات، وإن النقطة (A) تمثل مؤسسة غير

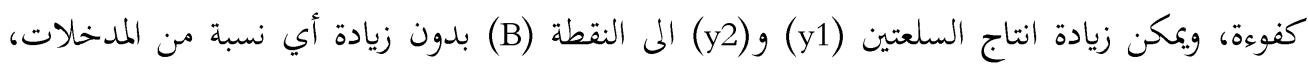
وتحسب الكفاءة الفنية لهذه المؤسسة على الشعاع (OC) عن طريق الصيغة الاتية:

$T E_{O}=\frac{O A}{O B}$

ويأخذ المؤشر القيم (1-0) وان القيمة (1) هي الكفاءة الفنية الكاملة للمؤسسة. 
وإن المستقيم (DD') يمثل السعر النسبي للمخرجات ويحسب مؤشر الكفاءة التوظيفية للمؤسسة التي تنتج عند النقطة (B) بدلا عن (B') على الشعاع (OC) ويكون بالصيغة الاتية: $A E_{O}=\frac{O B}{O C}$

وإن المسافة (BC) تمثل الزيادة في الايرادات التي يمكن تحقيقها بتوظيف المخرجات حسب المستوى(B') بدلا عن (B) (B). (1)

\section{صياغة نماذج تحليل مغلف البيانات:}

الصياغة الرياضية لتحليل مغلف البيانات:

يمكن حساب الكفاءة لـ (n) حسب مغلف البيانات من مؤسسات الأعمال التي لها مدخل واحد ومخرج واحد (بتال، 102:2012) وفقا للصيغة الاتية: j المخرج الفعلي للمؤسسة

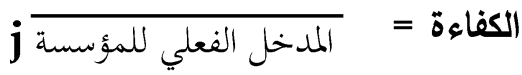

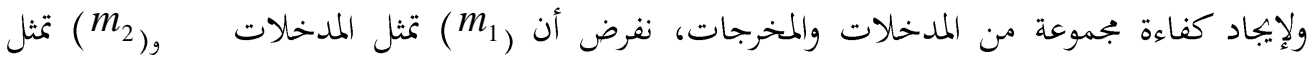
المخرجات، وستكون مصفوفة المدخلات والمخرجات وفق الصيغة الاتية:

$x_{i}=\left[\begin{array}{c}x_{1}^{j} \\ . . \\ x_{m 1}^{j}\end{array}\right], y^{j}=\left[\begin{array}{c}y^{j} \\ . . \\ y m_{2}\end{array}\right]: \mathrm{j}=1,2 \ldots \mathrm{n}$

إذ أن ( تمثل الأوزان للمدخلات والمخرجات على التوالي، ولحساب كفاءة المؤسسة

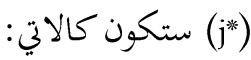

$E=\frac{\sigma_{1} y_{1}^{j^{*}} \ldots+\sigma_{m_{2}} y_{m_{2}}^{j^{*}}}{\pi_{1} x_{1}^{j^{*}} \ldots .+\pi_{m 1} x_{m_{1}}^{j^{*}}}$

$=\frac{\left(\sigma \cdot y^{j^{*}}\right)}{\left(\pi \cdot x^{j^{*}}\right)}$ 
وأنموذج البرججة الخطية الرياضي لإيجاد الكفاءة يكون وفق الصيغة الاتية (بهاء الدين، 2006: 95):

$\operatorname{Max} \frac{\left(\sigma \cdot y^{j^{*}}\right)}{\left(\pi \cdot x^{j^{*}}\right)}$

s. t.

$\frac{\left(\sigma \cdot y^{j^{*}}\right)}{\left(\pi \cdot x^{j^{*}}\right)} \leq 1: \mathrm{j}=1,2 \ldots \mathrm{n}$

وان الأنموذج (8-3) هو أنموذج برجمة كسرية يمكن تحويله إلى أنموذج برجة خطية يكون وفق الصيغة الاتية: $\operatorname{Max}\left(\sigma \cdot y^{j^{*}}\right)$

s. t.

$-\left(\pi \cdot x^{j^{*}}\right)+\left(6 \cdot y^{j^{*}}\right) \leq 0 \mathrm{j}=1,2 \ldots \mathrm{n}$

ويسمى بنموذج التوجيه الادخالي ذو عوائد الحجم الثابتة.

\section{النماذج الأساسية لتحليل مغلف البيانات}

هناك نماذج عديدة لإيجاد مؤشرات الكفاءة باستخدام أسلوب تحليل مغلف البيانات ومن أهمها أنموذج

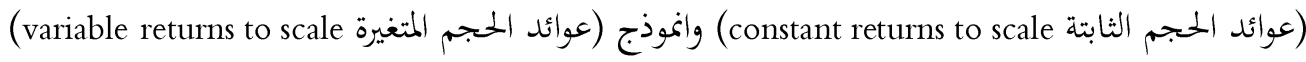
ويمكن ايجاد مؤشر الكفاءة للنموذجين عوائد الحجم الثابتة وعوائد الحجم المتغيرة، ومن جانب المدخلات تسمى (نماذج التوجيه الادخالي lnput oriented models) ومن جانب المخرجات تسمى (نماذج التوجيه الإخراجي output oriented models) (بتال، 2012: 103). أ- أنموذج عوائد الحجم الثابتة: وضع هذا الأنموذج كل من جارنز وكوبر وردوس (charnes, et al 1978)

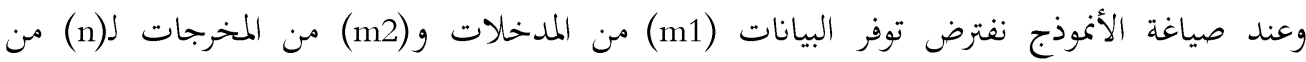

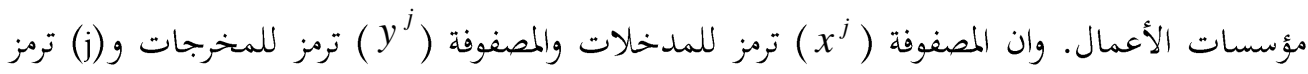
لمؤسسة. ويمكن حساب مؤشر الكفاءة للمؤسسة (ز3) باستخدام أنموذج التوجيه الادخالي / الإخراجي وتحل مسألة

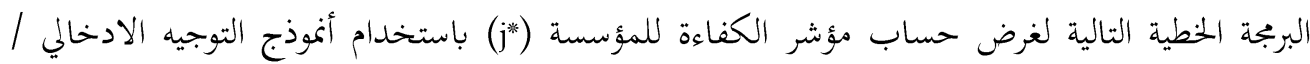
الإخراجي وتحل مسألة البربجة وفق الصيغة الاتية: 


\begin{tabular}{|l|l|}
\hline & \\
\hline $\max \left(\partial \cdot y^{j^{*}}\right)$ & $\min \left(\pi \cdot x^{j^{*}}\right)$ \\
s.t. & s.t. \\
$\left(\partial \cdot y^{j}\right)-\left(\pi \cdot x^{j}\right) \leq 0, j=1 \ldots . n:$ & $\left(\pi \cdot x^{j}\right)-\left(\partial \cdot y^{j}\right) \geq 0, j=1 \ldots . n:$ \\
$\left(\pi \cdot x^{j^{*}}\right)=1:$ & $\left(\partial \cdot y^{j^{*}}\right)=1:$ \\
$\pi, \partial \geq 0$. & $(1-13)$ \\
&
\end{tabular}

وباستخدام النموذج المقابل (duality) في البرجمة الخطية يمكن وضع الصيغتين السابقتين في الآي:

\begin{tabular}{|l|l|}
\hline & \\
\hline $\min \theta$ & $\max \phi$ \\
s.t & s.t \\
$\sum_{j} x^{j} \lambda_{j}-x^{j^{*}} \theta \leq 0, j=1 \ldots, n:$ & $\sum_{j} x^{j} \lambda_{j} \leq x^{j^{*}}, j=1 \ldots n:$ \\
$\sum_{j} y^{j} \lambda y_{j} \geq y^{j^{*}}:$ & $\sum^{\prime}$ التوجيه \\
$\lambda \geq 0$. & $\sum_{j} y^{j} \lambda_{j}-y^{j^{*}} \phi \geq 0:$ \\
$\lambda \geq 0$.
\end{tabular}

إن فرضية ثبات عائد الحجم تستخدم أنموذج مغلف البيانات عند عدم عمل كل المؤسسات عند مستوى الحجم الامثل مما يؤدي الى خلط مؤشرات الكفاءة الفنية بالحجمية وعند الفصل بين أثر الكفاءة الفنية والحجمية تستخدم خاصية تغير عائد الحجم للإنتاج ويكون (ثابت، متزايد أو متناقص) مما يعني ان الزيادة في مدخلات المؤسسة بنسب معينة قد تؤدي إلى زيادة كبيرة أو قليلة أو متساوية في الانتاج.

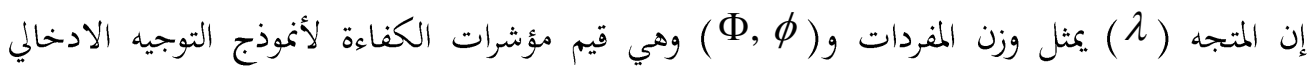

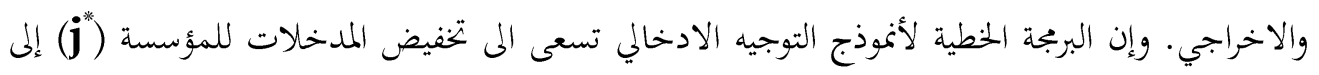

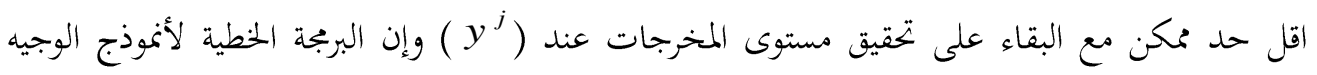

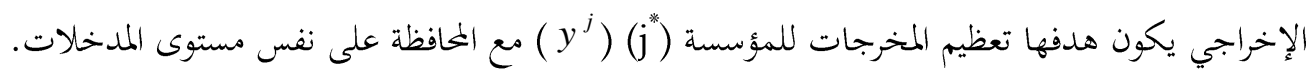
وإن هذا الأنموذج يعتمد على خاصية ثبات عائد الحجم للانتاج، اي ان تغير كمية المدخلات المستخدمة

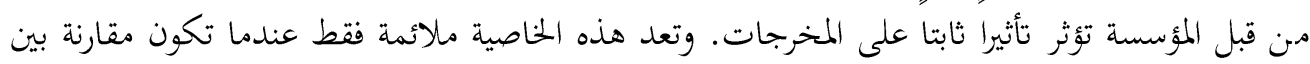
جميع المؤسسات التي تعمل بمستوى أحجامها المثلى. ولكن وفي بعض تلمض الأحيان توجد عوائق كثيرة تمنع المؤسسات من تحقيق هذه الأحجام مثل قيود التمويل والمنافسة غير التامة (بابكر، 22:2002) 
ب-أنموذج عوائد الحجم المتغيرة: إن هذا الأنموذج يميز بين نوعين من الكفاءة وهي الكفاءة الفنية والكفاءة الحجمية، ووضع هذا النموذج من قبل بانكر، شارنز وكوبر (Banker et al 1984). ويعدل نموذج عوائد الحجم الثابتة إلى نموذج عوائد الحجم المتغيرة في البرجمة الخطية عن طريق اضافة متغير منفصل وهو ( E) )، لمعرفة صفة عوائد الحجم المتغيرة للمؤسسات المصرفية، ويمكن صياغة أنموذج البربجة

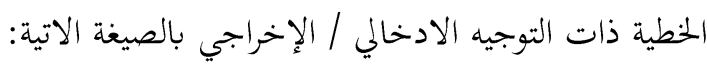

\begin{tabular}{|l|l|}
\hline & \\
\hline $\max \left(\partial \cdot y^{j^{*}}\right)+\varepsilon$ & $\min \left(\pi \cdot x^{j^{*}}\right)+\varepsilon$ \\
s.t. & s.t. \\
$\left(\partial \cdot y^{j}\right)-\left(\pi \cdot x^{j}\right)+\varepsilon \leq 0, j=1 \ldots . n$ & $\left(\pi \cdot x^{j}\right)-\left(\partial \cdot y^{j}\right)+\varepsilon \geq 0, j=1 \ldots . n:$ \\
$\left(\pi \cdot x^{j^{j *}}\right)=1:$ & $\left(\partial \cdot y^{j^{*}}\right)=1:$ \\
$\pi, \partial \geq 0$. & $\pi, \partial \geq 0$. \\
& \\
\hline
\end{tabular}

وعند استخدام النموذج المقابل يمكن كتابة المسألتين السابقتين بالصيغة الاتية:

\begin{tabular}{|c|c|}
\hline التوجيه الادخالي & التوجيه الاخراجي \\
\hline $\min \theta$ & $\max \phi$ \\
\hline s.t & s.t \\
\hline$\sum_{j} x^{j} \lambda_{j}-x^{j^{*}} \leq 0, j=1 \ldots, n:$ & $\sum j x^{j} y_{j} \leq x^{j}, j=1 \ldots n$ \\
\hline$\sum_{j} y^{j} \lambda_{j} \geq y^{j^{*}}:$ & $\sum j y^{j} y_{j}-y^{j^{*}} \phi \geq 0:$ \\
\hline$\sum_{j} \lambda_{j}=1:$ & $y_{j} \geq 0$ \\
\hline$\lambda \geq 0$. & \\
\hline
\end{tabular}

الشكل (3-5) الحدود المثلى للإنتاج لنماذج (VRS , CRS) ومواقع مجموعة من المؤسسات لدالة انتاجية بسيطة تحتوي على يخرج واحد (y)ومدخل واحد (X). 
شكل (1-1) عوائد الحجم ونماذج DEA

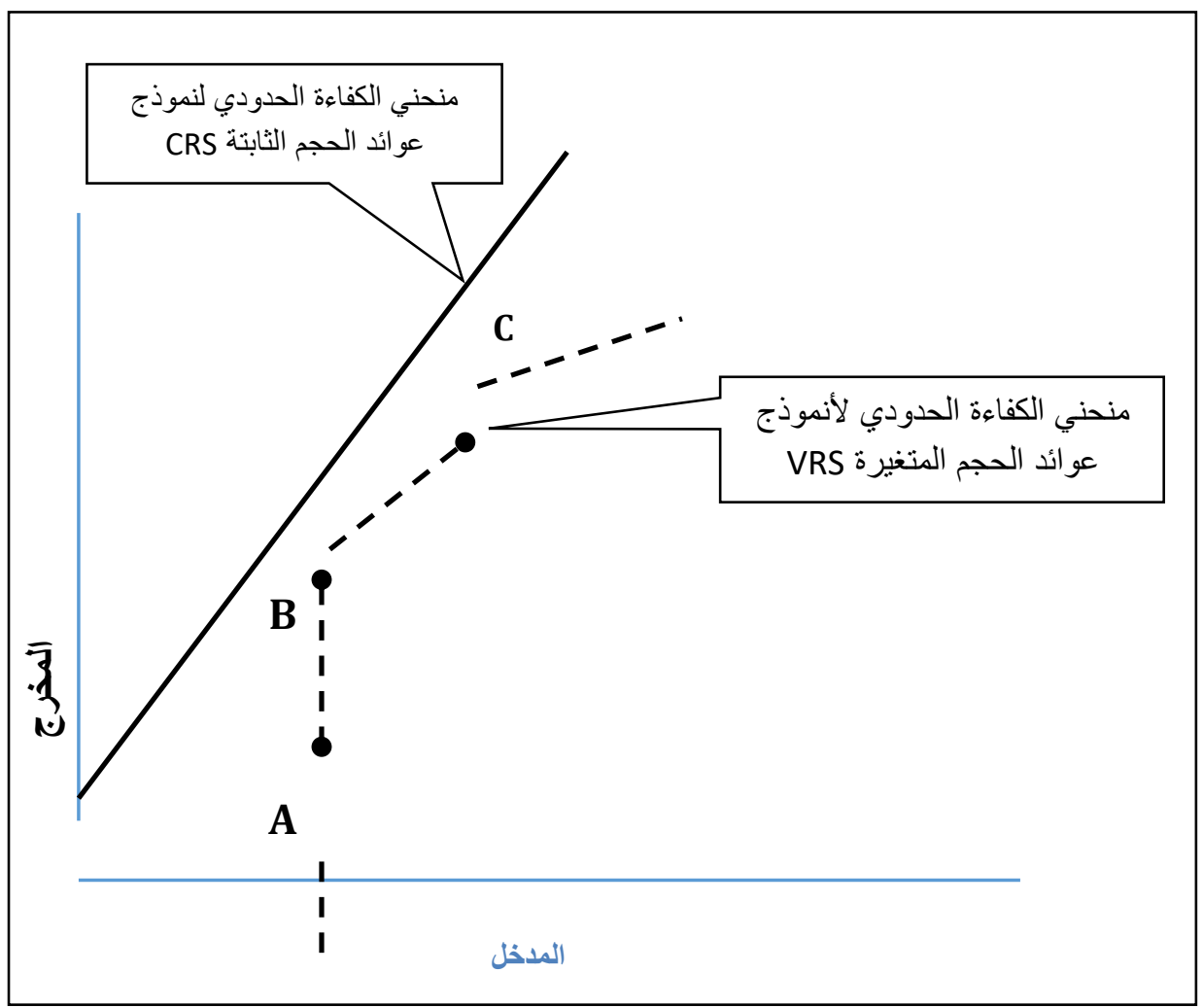

المصدر: بتال، احمد حسين، قياس وتحليل كفاءة أداء المصارف الخاصة في العراق باستخدام تكنيك تحليل مغلف البيانات، اطروحة

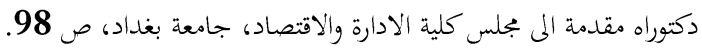

يوضح الشكل (1-5) بأن المؤسسات (A,B,C) هي مؤسسات كفوءة من الناحية الفنية لعملها على منحنى الكفاءة الحدودي، وهذه المؤسسات كفوءة من الناحية الفنية إلا أها غير متساوية من الناحية

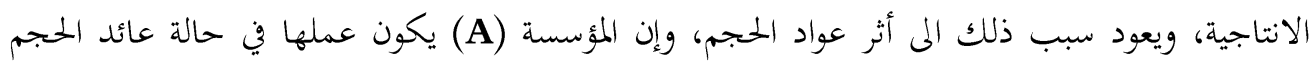
المتزايد اي أها لم تصل الى مستوى انتاجها الامثل بالنسبة لحجمها وتستطيع ان تحقق انتاجية اكبر من إنس

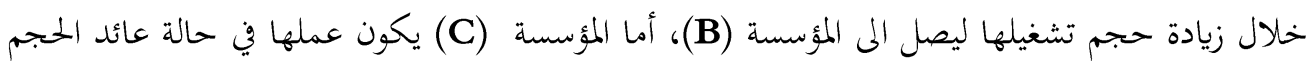
ذالمتناقص على منحنى الانتاج الحدودي، فينبغي أن تقلل حجم عملياتا حتى تصل الى المؤسسة (B) لتصبح ذات انتاجية اكبر، ونجد بأن المؤسسة (B) يكون عملها عند افضل مستوى من الانتاج ( Coelli etal, ) (2005:59 


\section{مجالات ومزايا تطبيق أسلوب تحليل مغلف البيانات والدور والتحفظات التي يؤديها.}

\section{مجالات تطبيق أسلوب مغلف البيانات:}

بالرغم من أن أسلوب مغلف البيانات لم يعرف إلا عام (1978م)، وان الاهتمام الكبير بأسلوب تحليل مغلف البيانات من قبل الممارسين والأكاديمين بدأ من خلال الأبحاث الكثيرة التي الجزت، فقد شمثلت هذه الابحاث معظم الجمالات والانشطة الاقتصادية والمالية ولاسيما القطاع المصرفي والقطاع الصناعي التي اعتمدت مغلف البيانات في دول العالم كافة (Charnes,A,Cooper,1994:212) أما في الوطن العربي فيعد أسلوب تحليل مغلف البيانات حديثا والدراسات المعدة وفق هذا الأسلوب تكاد تكون قليلة إذا ماقورنت

$$
\text { مع بقية دول العالم. }
$$

أهم مزايا تحليل مغلف البيانات:

يعد أسلوب تحليل مغلف البيانات اداة فعالة لقياس الكفاءة في كافة القطاعات سواء كانت عامة أو خاصة، ومن الصعب لهذا القطاع تحسين عملياته من خلال الضغط على التكاليف دون التضحية بنوعية الخدمات المقدمة بسبب وجود عوامل عديدة تؤثر في نوعية الانتاجية والخدمة، ومن الصفات المميزة لهذا الاسلوب هي عدم حاجة مغلف البيانات الى وضع أية فرضيات للدالة التي تربط بين المتغيرات التابعة

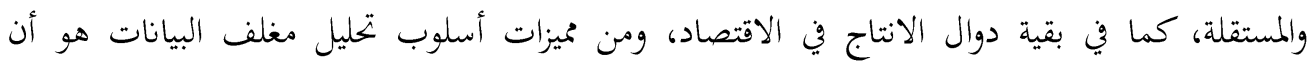
البيانات تتحدث لنفسها بدلا من أن تتحدث في إطار صيغة دالة مفروضة عليها أي عدم وجود اي فرضيات لأسلوب تحليل مغلف البيانات وهناك بعض المميزات والسلبيات التي توجه لهذا الأنموذج وهي (Charnes, A,Cooper,1994:212). 1-1إنه أسلوب سهل الاستخدام لذلك يستخدم بشكل واسع خصوصاً في تحليل كفاءة المصارف، ويمتاز أسلوب مغلف البيانات بأنه عند حساب الكفاءة يأخذ بالاعتبار فقط المؤسسات الاكثر كفاءة عند تشكيل منحنى الكفاءة الحدودي.

2-إن أسلوب تحليل مغلف البيانات لا يحتاج الى صيغة دالة معينة عند الشروع باستخدامه في قياس الكفاءة الانتاجية، ولا يحتاج مغلف البيانات الم افتراضات مسبقة عن شكل العلاقة بين المدخلات والمخرجات. 3-أهم الانتقادات الموجهة على تطبيق أسلوب تحليل مغلف البيانات هو عدم القدرة على التمييز بين نقص الكفاءة والخطأ الاحصائي، كذلك هو أسلوب حساس لعدد المتغيرات الداخلة للنموذج أي كلما إزداد عدد المتغيرات الداخلة كلما ازدادت الوحدات الكفوءة. 
4-ومن سلبياته بأنه يتبنى مفهوم الأوزان لكل مؤسسة عند تعظيم الكفاءة النسبية لها, فقد تظهر المؤسسة

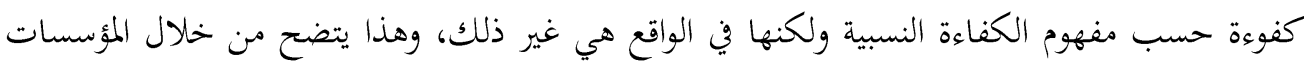
الداخلة في التقييم إذا كانت قليلة وعدد المخرجات كبير.

\section{المبحث الثاني خطوات تطبيق نموذج DEA على المصارف الحكومية.}

\section{مثغيرات الدراسة (المدخلات والمخرجات).}

يعد اختيار المدخلات والمخرجات مرتكزا هاما في تطبيق أسلوب مغلف البيانات، لأن ذلك يؤثر في تفسير واستخدام النتائج، لذا لابد من توافر بعض الشروط في اختيارها، ولعل أهمها هي: (Avkiran,1999:221) 1- وجود علاقة تربط بين المدخلات والمخرجات.

2- إن تكون المدخلات والمخرجات تتسم بالشمولية، بمعنى أن يكون لديها القدرة على أن تعكس أنشطة الوحدة التي سيتم تقييم ادائها. بمعنى ان المدخلات والمخرجات أدوات مشتركة في المصارف الحكومية. 3- أن تكون البيانات دقيقة معرضة للتدقيق من قبل الجهات العليا. كما أن الدراسات السابقة تختلف في دراستها في اختيار المدخلات والمخرجات تبعا للبيانات إلا أن هناك طرق تساعد على تحديد المدخلات والمخرجات وهي: 1-عرض متغيرات الدراسة التي تم اختيارها على مجموعة من أساتذة الاقتصاد والإدارة المالية. 2-إن المصارف الحكومية بحارية تتقارب في هدفها الى تمقيق أقصى موجودات بهدف زئيات زيادة العائد ومنح أكبر قرض ممكن، وتتقارب في مدخلاتها لرأس المال. واستناداً لبعض الدراسات التطبيقية لقياس كفاءة المصارف، نجد أنسارت أهم المخرجات تشمل الموجودات والائتمان الممنوح والتي هي الخصلة النهائية للمصرف. أما المدخلات فهي كل متغير له علاقة بزيادة الموجودات والائتمان، وبناءاً على البيانات التي يمكن الحصول عليها، يمكننا تحديد المدخلات والمخرجات فئه للنموذج الذي سوف يتم استخدامه في قياس كفاءة أداء المصارف، وكما في الشكل الآتي: 


$$
\text { الشكل (1-6) المدخلات والمخرجات للمصارف الحكومية }
$$

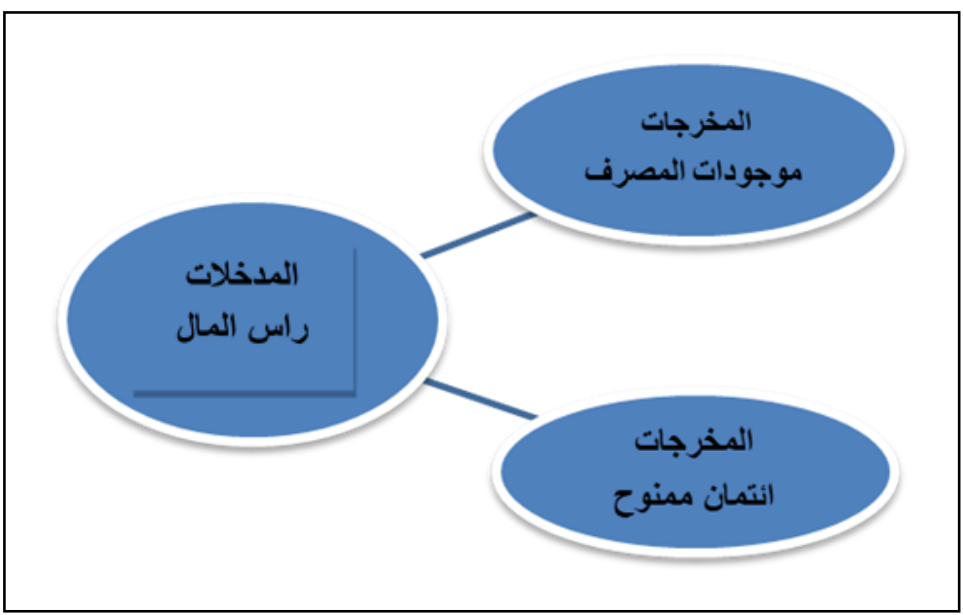

المصدر: اعداد الباحث بالاعتماد على الجداول (2-2 و2-3و 2-4)

\section{تحديد خيارات التحليل}

تعظيم المخرجات (Output Max) أو تخفيض المدخلات (Input Min)، فقد أختار الباحث تعظيم المخرجات، لأن (Avkiran,1999:211) يرى أن أنموذج (BBC) أفضل من أنموذج (CCR) لان تعظيم المخرجات يتطلب تحليله بشكل اوسع من المدخلات، عندما يكون التوسع في حجم العمليات مطلوبا، في حين يلجأ إلى تخفيض المدخلات في حالة الانكماش الاقتصادي وضرورة تخفيض حجم العمليات. فقد

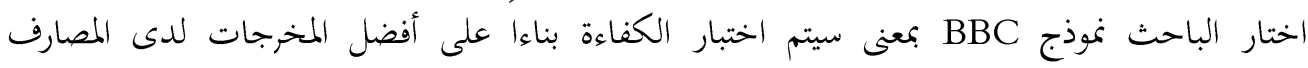
المكومية.

\section{إختيار عينة البحث والوصف الإحصائي للمتغيرات:}

\section{إختيار عينة البحث:}

إن المصارف العراقية المختارة هي (7) مصارف حكومية، وتعد من المصارف الفعالة في العراق ولديها عمق وتداول مالي واسع. كما ان هذه العينة موافقة لشروط الاختيار التي يتوافر فيها إمكانية تطبيق تحليل مغلف كانف

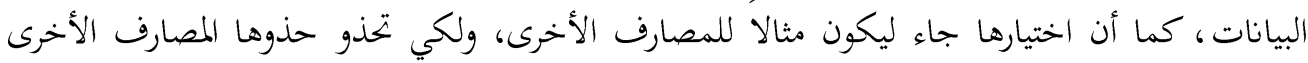

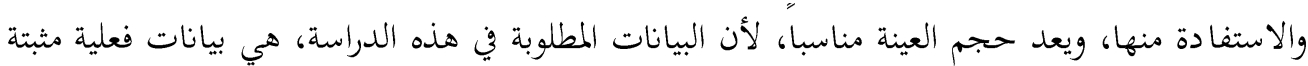
ومدققة. وتم الحصول عليها من: 


$$
\text { 1 - 1 تقارير الاستقرار المالي للبنك المركزي العراقي. }
$$

2- النشرات الاحصائية السنوية للبنك المركزي العراقي.

3- تقارير المصارف العراقية السنوية.

فكانت العينة كما يمثلها الجدولين الآتيين:

الجحدول (1-1) متوسط المدخلات والمخرجات للمصارف الحكومية للمدة (2010- 2019) مليون دينار

\begin{tabular}{|c|c|c|c|}
\hline \multicolumn{2}{|c|}{ المخرجات } & المدخلات & \\
\hline الائتمان النقدي & الموجودات & راس المال المصرف & \\
\hline 12644595 & 100553569 & 95600 & مصرف الرافدين \\
\hline 6303291 & 43427427 & 30800 & مصرف الرشيد \\
\hline 3082493 & 2502848 & 75600 & المصرف الزراعي \\
\hline 198462.2 & 724316 & 145000 & المصرف الصناعي \\
\hline 3211777 & 2443157 & 50000 & المصرف العقاري \\
\hline 5574049 & 27946639 & 1575000 & المصرف العراقي للتجارة \\
\hline 11261 & 101988 & 52900 & مصرف النهرين الإسلامي \\
\hline
\end{tabular}

المصدر: النشرة الاحصائية السنوية للبنك المركزي العراقي (2010- 2019)، دائرة الاحصاء والابحاث، اعداد مختلفة

\section{الوصف الإحصائي للمتغيرات (للمدخلات والمخرجات)}

يوضح الجدول (1-2) الانحراف المعياري لكل مدخل ومخرج للمصارف الحكومية، ويبين الجدول بأن هناك إنخفاض وإرتفاع للانخراف المعياري للمصارف، كما يبين الجدول (1-1) معامل الارتباط للمدخلات وعلاقتها بالمخرج، فيوضح أن معامل أرتباط قوي لبعض المصارف وضعيف بالنسبة لمصارف أخرى، ورغم

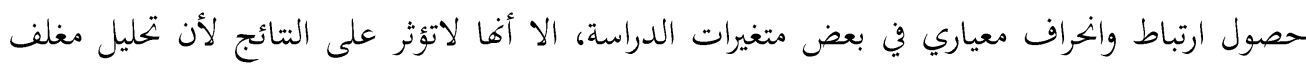
البيانات هو نموذج غير معلمي. 
جدول (1-2) الانخراف المعياري بين المثغيرات (المدخلات والمخرجات)

\begin{tabular}{|c|c|}
\hline الاخراف المعياري & المصرف \\
\hline 54737660 & مصرف الرافدين \\
\hline 23454967 & مصرف الرشيد \\
\hline 1595250 & المصرف الزراعي \\
\hline 320152.9 & المصرف الصناعي \\
\hline 1648980 & المصرف العقاري \\
\hline 14212603 & المصرف العراقي للتجارة \\
\hline 45414.44 & مصرف النهرين الإسلامي \\
\hline
\end{tabular}

(DEAP) المصدر : بالاعتماد على مخرجات

\begin{tabular}{|c|c|c|}
\hline الائتمان النقدي & الموجودات & رأس المال \\
\hline 0.96038 & 0.031465 & 1 \\
\hline 0.110173 & 1 & 0.031464791 \\
\hline 1 & 0.110173 & 0.960380361 \\
\hline
\end{tabular}

(DEAP) المصدر: بالاعتماد على مخرجات

تطبيق تحليل (DEA) على المؤشرات للمصارف الحكومية:

لغرض قياس الكفاءة للمصارف الحكومية، باستخدام تحليل مغلف البيانات، قمنا بتوظيف برنامج ( DEAP Version 2.1 2019)، من خلال استخدام نموذج عوائد الحجم المتغيرة وبالتوجه المخرجي فقط. يبين الجدول (1-4) تقدير الكفاءة (1) لمتوسط المؤشرات المالية للمصارف الحكومية للمدة -2019

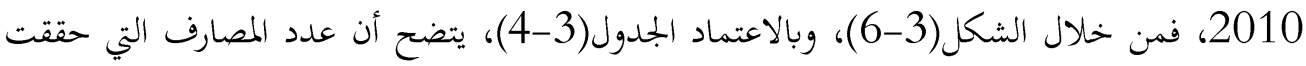
كفاءة (1)، في المصارف عدد (1)، وهو مصرف النهرين الإسلامي فيماكانت المصارف غير الكفؤة والتي حققت كفاءة أقل من (1) هي مصارف (الرافدين، الرشيد، الزراعي، الصناعي، العقاري، العراقي للتجارة) 
على التوالي، كما يوضح الجدول (1-4) النقص الحاصل في الكفاءة، والمصرف الذي يتطلب كفاءة مرتفعة

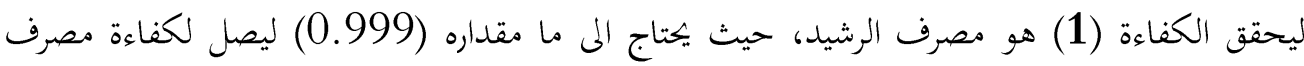
النهرين الإسلامي.

\begin{tabular}{|c|c|c|}
\hline نقص الكفاءة & مؤشر الكفاءة & المصارف الحكومية \\
\hline 0.998 & 0.002 & مصرف الرافدين \\
\hline 0.999 & 0.001 & مصرف الرشيد \\
\hline 0.942 & 0.058 & المصرف الزراعي \\
\hline 0.614 & 0.386 & المصرف الصناعي \\
\hline 0.961 & 0.039 & المصرف العقاري \\
\hline 0.891 & 0.109 & المصرف العراقي للتجارة \\
\hline 0 & 1.000 & مصرف النهرين الإسلامي \\
\hline 0.772 & 0.228 & متوسط الكفاءة \\
\hline
\end{tabular}

(DEAP) المصدر: بالاعتماد على مخرجات

الشكل (1-7) مؤشر نقص الكفاءة (1) للمصارف الحكومية

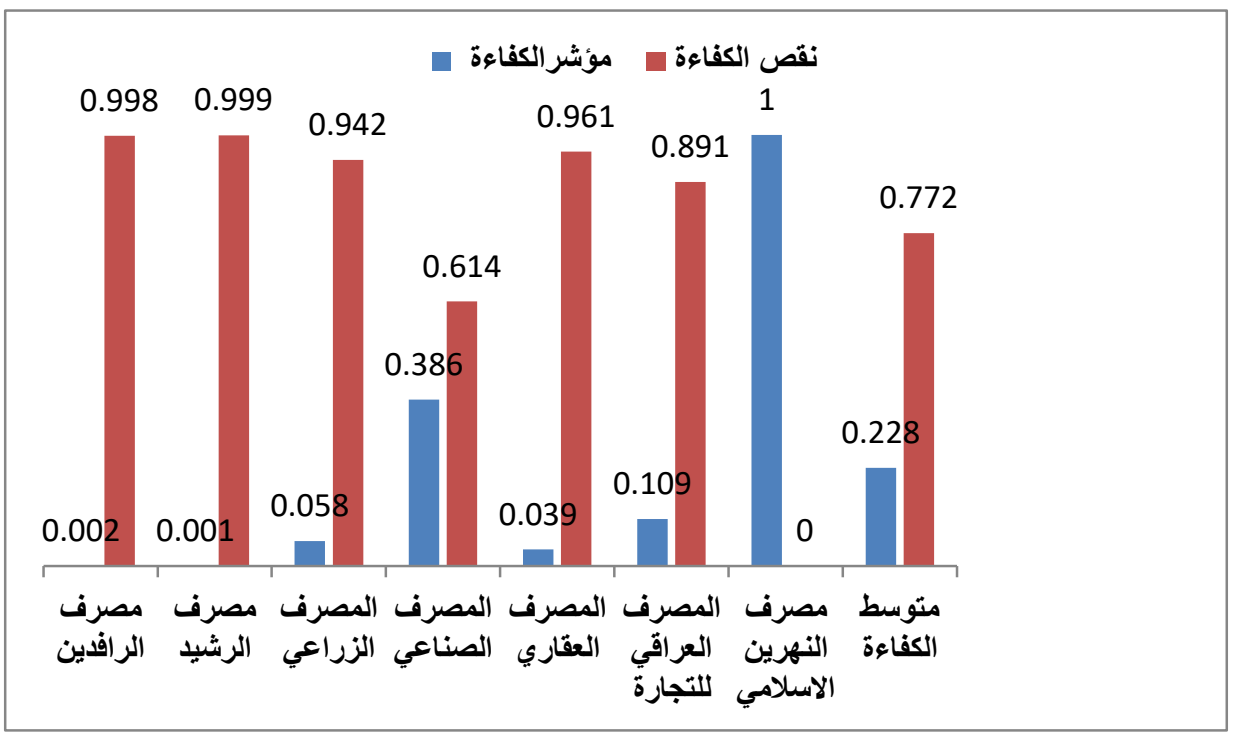

المصدر: بالاعتماد على الجلدول (1 - 4) 
إن المصارف الكفوءة شكلت ما يسمى (بالحزام الأمامي للكفاءة) والمصارف غير الكفوءة تقع أدنى ذلك

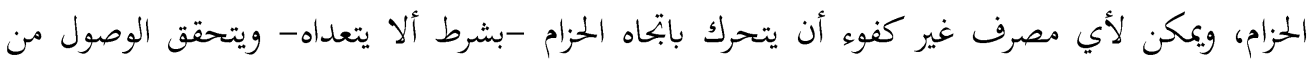

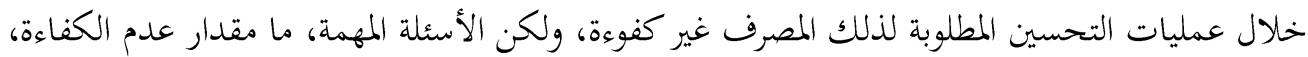
ومن هي المصارف المرجعية للمصارف غير الكفوهة، وما كمية المخرجات الراكدة؟ سيتم الإجابة على تلك الأسئلة، للوصول إلى التحسينات المطلوبة للمصارف غير الكفوءة. لذلك إذا أرادت المصارف من رفع كفاءقا وتساهم في عملية التنمية الاقتصادية عليها أن تستغل الموارد الراكدة غير المستغلة فنلاحظ من الجمدول (1-5) حجم الموارد غير المستغلة من الائتمان النقدي والذي يفيض عن الحاجة والمتراكم داخل المصرف فمن الممكن استخدامه في عمليات الاستثمار وتفعيل المخرجات بأقصى قدر بمكن، فمثنلا مصرف الرافدين لابد ان ان يستغل المخرج الراكد الموجود والبالغ (1541977.628) ودخوله في دورة الاستثمار .

\begin{tabular}{|c|c|c|}
\hline حجم الائتمان النقدي الراكد & حجم الموجودات الراكدة & المصارف الحكومية \\
\hline 1541977.628 & 0.000 & مصرف الرافدين \\
\hline 1508253.785 & 0.000 & مصرف الرشيد \\
\hline 2806141.161 & 0.000 & المصرف الزراعي \\
\hline 118486.885 & 0.000 & المصرف الصناعي \\
\hline 2942015.940 & 0.000 & المصرف العقاري \\
\hline 2488322.230 & 0.000 & المصرف العراقي للتجارة \\
\hline 0.000 & 0.000 & مصرف النهرين الاسلامي \\
\hline 1629313.947 & 0.000 & متوسط الراكد \\
\hline
\end{tabular}

المصدر : بالاعتماد على خخرجات (DEAP) 
الشكل (1-8) حجم الراكد للمصارف الحكومية

حجم الايتمان النقاي الراكد ص حجم الموجودات الراكدة

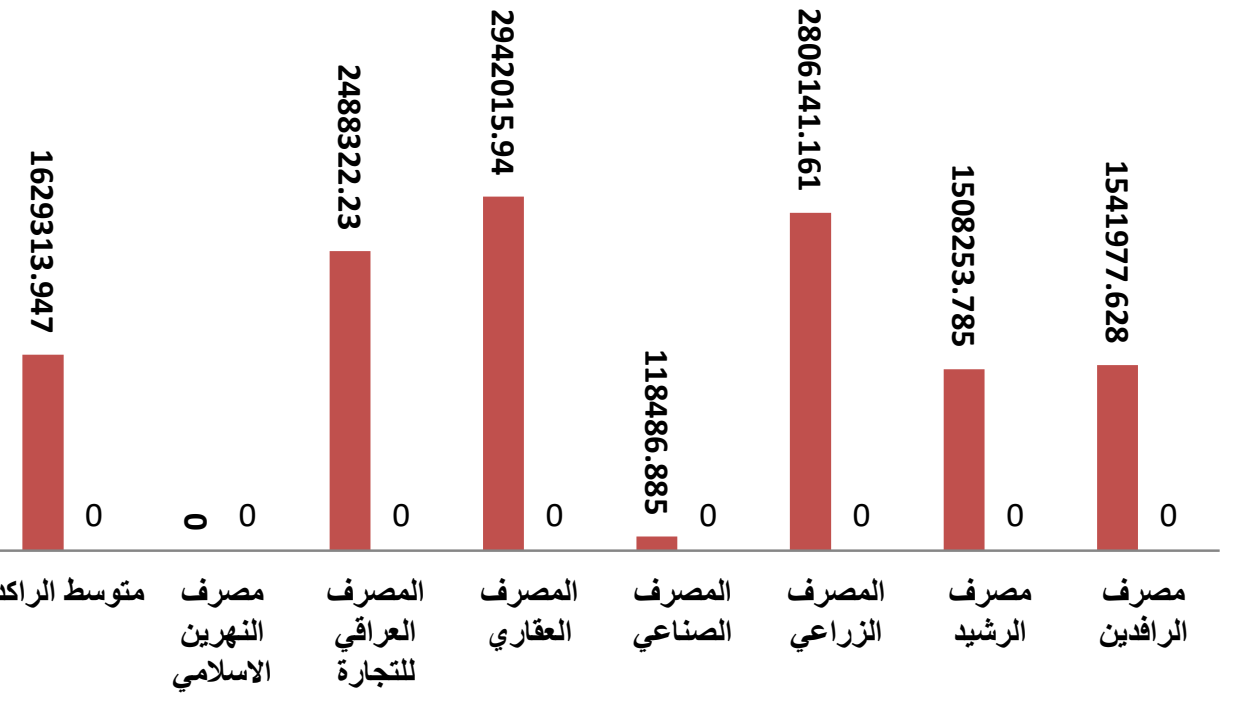

المصدر: بالاعتماد على الجدول (1-5) (5)

اما المدخلات الراكدة في المصارف فأها لا تمتلك أي مدخلات راكدة وكما يمثلها الجدول والشكل الاتي:

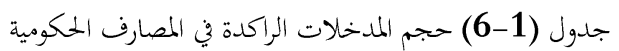

\begin{tabular}{|c|c|}
\hline حجم المدخحلات الراكدة & لمصارف الحكومية \\
\hline 0.000 & مصرف الرافدين \\
\hline 0.000 & مصرف الرشيد \\
\hline 0.000 & المصرف الزراعي \\
\hline 0.000 & المصرف الصناعي \\
\hline 0.000 & المصرف العقاري \\
\hline 0.000 & لمصرف العراقي للتجارة \\
\hline 0.000 & مصرف النهرين الإسلامي \\
\hline 0.000 & متوسط الراكد. \\
\hline
\end{tabular}

المصدر: بالاعتماد على خرجات (DEAP) 
لهذا فان المصارف التي لم تحقق كفاءة (1) فان المرجع لها هو مصرف النهرين الإسلامي الذي تساوت مدخلاته مع مخرجاته وهو يعد مثالا لبقية المصارف الأخرى، وكما يمثلها الجدول الآني:

جدول (1-7) المصارف المرجعية

\begin{tabular}{|c|c|c|}
\hline حجم المخرجات الراكدة & المصارف الحكومية & ت \\
\hline 7 & مصرف الرافدين & 1 \\
\hline 7 & مصرف الرشيد & 2 \\
\hline 7 & المصرف الزراعي & 3 \\
\hline 7 & المصرف الصناعي & 4 \\
\hline 7 & المصرف العقاري & 5 \\
\hline 7 & المصرف العراقي للتجارة & 6 \\
\hline & مصرف النهرين الاسلامي & 7 \\
\hline
\end{tabular}

المصدر : بالاعتماد على خرجات (DEAP)

يمكن للمصارف أن تستعيد كفاءتا إذا حققت الأهداف المطلوبة منها وعلى مستوى كل مصرف فمثلا مصرف الرافدين يهدف الى تعظيم مدخلاته بحدود (52155977.175) وأن يهدف الى تخفيض مخرجاته من الائتمان الى (11102617.372) وهكذا لبقية المصارف، وكما في الجدول (1-8).

جدول (1-8) الحجم المستهدف من المدخلات والمخرجات

\begin{tabular}{|c|c|c|c|}
\hline الائتمان & الموجودات & المخرجات المستهد من المدخلات & المصارف الحكومية \\
\hline 11102617.37 & & 52155977.175 & مصرف الرافدين \\
\hline 4795037.215 & 43427427 & 22525305.804 & مصرف الرشيد \\
\hline 276351.839 & 2502848 & 1298198.408 & المصرف الزراعي \\
\hline 79975.315 & 724316 & 375694.360 & المصرف الصناعي \\
\hline 269761.060 & 2443157 & 1267237.374 & المصرف العقاري \\
\hline 3085726.770 & 27946639 & 14495599.513 & المصرف العراقي للتجارة \\
\hline 11261.000 & 101988 & 52900.000 & مصرف النهرين الاسلامي \\
\hline
\end{tabular}

المصدر : بالاعتماد على خرجات (DEAP) 
إن هذا البحث يتفق مع العديد من الدراسات، ومنها دراسة (Căpraru \& Marius) التي بحثت في تأثير

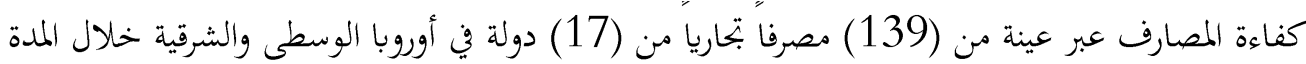

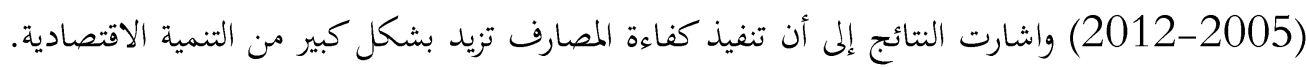
وأوضحت ان الكفاءة المصرفية تزيد من النمو الاقتصادي ولاسيما ذات أسات أس المال المال الكبير.

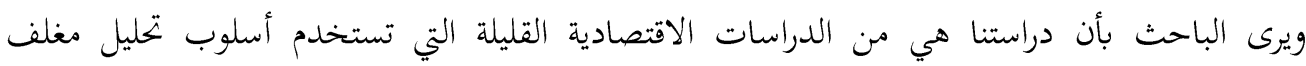

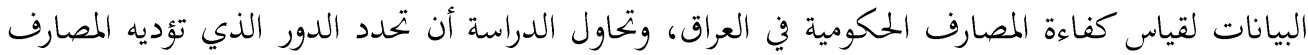

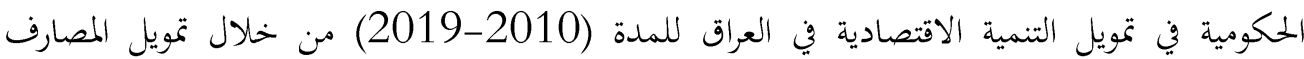
للقطاعات الاقتصادية كافة مما يسهم بتحقيق عملية التنمية الاقتصادية، وإن مفهوم الكفاءة المصرفية

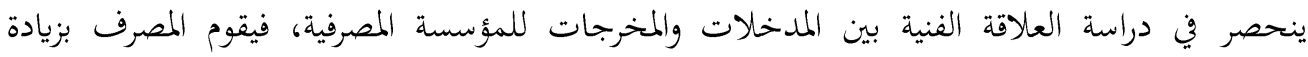

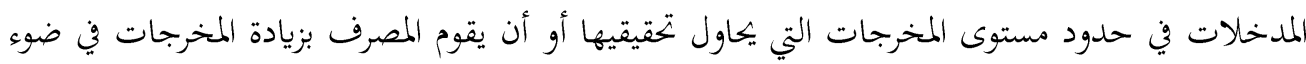

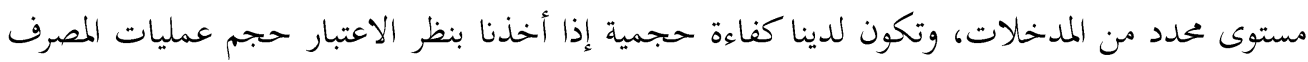

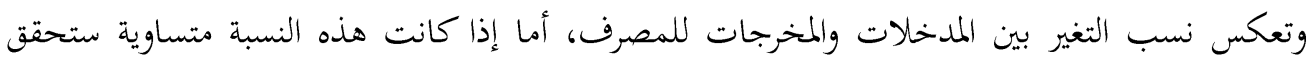

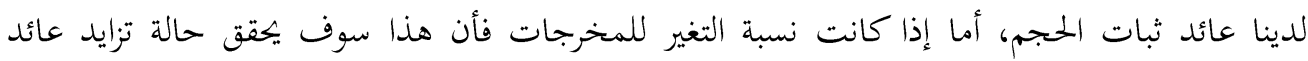

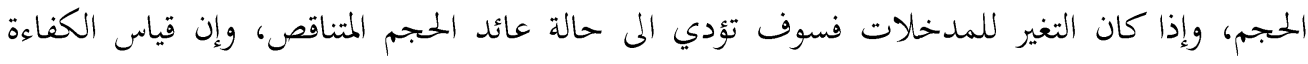

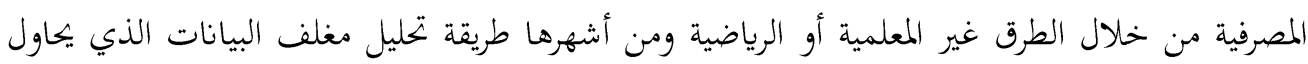

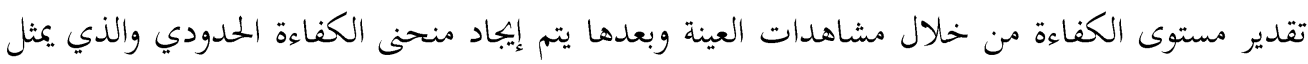

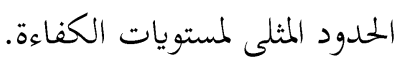

الخاتقة

\section{الاستنتاجات}

1- إن عدد المصارف التي حققت كفاءة (1) هو مصرف النهرين الإسلامي، فيما كانت المصارف غير التاني

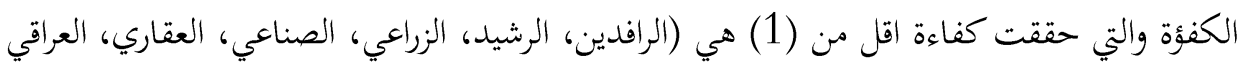
للتجارة)

2- بينت الدراسة حجم المخرجات الراكدة من الموجودات والائتمان النقدي لدى المصارف، فمثلا

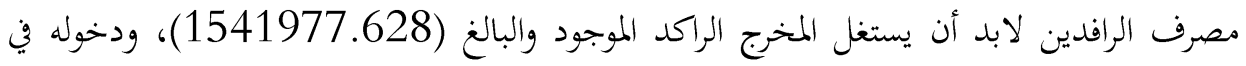


دورة الاستثمار، كما بينت الدراسة انه لا يوجد مدخلات غير مستغلة في المصارف والمتمثلة براس المال.

3- أوضحت الدراسة أن جميع المصارف إذا ارادت أن تحقق الكفاءة عليها الرجوع الى المصرف المرجعي

$$
\text { وهو مصرف النهرين الإسلامي. }
$$

4- بلغ متوسط الكفاءة المصرفية لجميع المصارف خلال المدة (2010-2019) بمقدار (0.228) وهو مقدار متدني حيث أنه يحتاج الى تحسين بمقدار (0.772).

\section{التوصيات}

1- أن تقوم المصارف التي لم تحقق الكفاءة النسبية المطلوبة بدراسة الأسباب التي أدت بالمصارف المرجعية

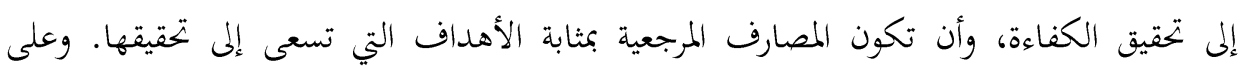

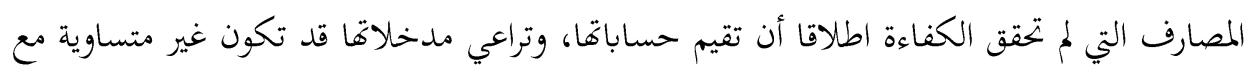
مخرجاةًا. 2- العمل على زيادة دور التكنولوجيا المصرفية الحديثة التي توفر الأساليب والوسائل التي من شأها رفع

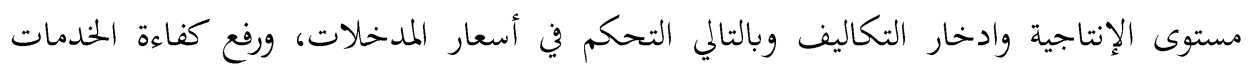

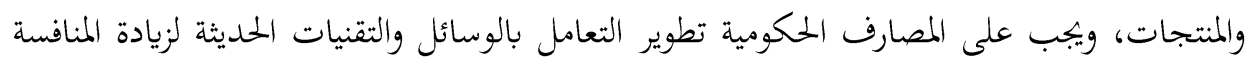
بين المصارف الحكومية. 3- ان تقوم المصارف بإجراء دراسات مستقبلية باستخدام أسلوب تحليل مغلف البيانات تأخذ بنظر الاعتبار المقارنة بين كفاءة المصارف الحكومية والمصارف الخاصة في العراق، أو عمل مقارنة بين كفاءة المصارف العراقية مع المصارف العربية أو الاجنبية.

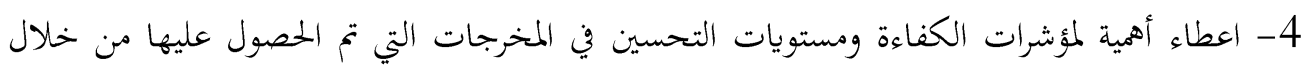

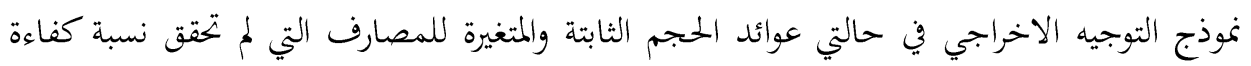
(100\%) وإن المصارف غير الكفوءة عليها أن تستفيد من أنماط التشغيل والخبرات التي تمتلكها المصارف المرجعية التي استطاعت تحقيق كفاءة تامة. 
3. بهاء الدين، محمد شامل، (2006)، استخدام اسلوب مغلف البيانات في قياس الكفاءة النسبية للوحدات الادارية: دراسة احصائية - تطبيقية مجلة

$$
2 \text { النهضة - مصر، مجلد:7، العداء } 2
$$

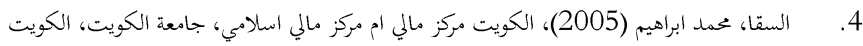

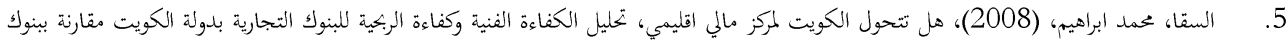

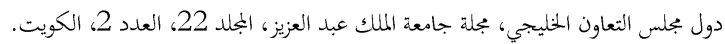

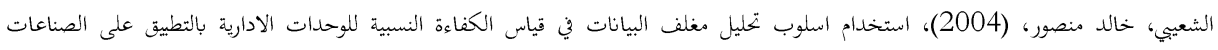

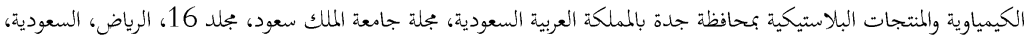
7. 8. منصوري، عبد الكريم، (2014)، قياس الكفاءة النسبية ومحدداها للانظمة الصحية باستخدام تحليل مغلف البيانات للبلدان المتوسطة والمرتفعة الدخل، اطروحة دكتوراه، غير منشورة، جامعة ابي بكر بلقايد - الجزائر.

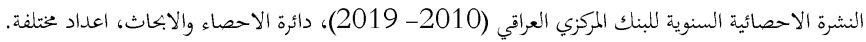

